\title{
Design and Analysis of a Novel CPT System with Soft Ferromagnetic Material Cores and Electromagnetic Resonant Coupling for EVs
}

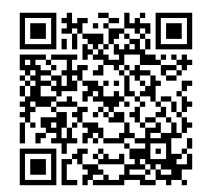

\author{
Junlong Duan ${ }^{1 *}$ and Weiji Wang ${ }^{2}$ \\ ${ }^{1}$ Department of Engineering and Design University of Sussex, UK \\ ${ }^{2}$ Department of Engineering and Design University of Sussex, UK
}

Submitted: July 31, 2019; Published: August 30, 2019

*Corresponding author: Junlong Duan, Department of Engineering and Design University of Sussex, Brighton BN1 9QT, United Kingdom

\begin{abstract}
This paper describes a novel contactless power transfer (CPT) system with geometrically improved H-shape ferromagnetic cores and electromagnetically prospective modelling analysis methods for wireless power transmitting (WPT) applications of electric vehicles (EVs). A CPT prototype, using optimized H-shaped magnetic couplers and series-to-series (SS) compensation, is proposed to address and ensure the maximization of system efficiency, power transfer ratings, and air gaps of coupling coils. By focusing on the main factors such as various system operating frequencies, different geometric designs of coils, changeable inductive coupling distances, electromagnetic field performances and actual phase angle deviations when the inductive coupling system tends to be stable with its waveforms, this small-sized H-shape CPT system has been analytically considered and modelled in a finite-element method (FEM) environment, resulting in a maximum system efficiency of $59.5 \%$, a coil transmitting efficiency of $83.8 \%$ and a maximum power output of $42.81 \mathrm{~kW}$ on the load end when the resonant coupling of CPT system tends to occur within a range of calculated resonant frequencies, with an air gap of $10 \mathrm{~mm}$. Moreover, the system efficiency and coil transmitting efficiency can reach $47.75 \%$ and $77.22 \%$, respectively, and the highest RMS real power to load can achieve $31.95 \mathrm{~kW}$ with an air gap of $20 \mathrm{~mm}$. Besides, with an air gap of $30 \mathrm{~mm}$, this H-shape CPT system is measured to output 20.39-kW RMS power, along with the maximum system efficiency and coil efficiency of $41.78 \%$ and $63.23 \%$, respectively. Furthermore, the improvements of flux linkage, magnetic flux density regarding the actual electromagnetic performance produced and the issues on the calculated natural resonant frequencies have been studied by result analysis and comparison of electromagnetic field parameters generated. In addition, the current limitations and further design considerations have been discussed in this paper.
\end{abstract}

Keywords: Contactless power transfer; Wireless power transmitting; Magnetic resonant coupling; Inductive power transfer; Ferrite, Electric vehicles; Charging efficiency; Finite element method; Maxwell equations; Ferromagnetic; Soft magnetic material

Abbreviations: CPT: Contactless power transfer; WPT: wireless power transmitting; MRC: magnetic resonant coupling; IPT: Inductive Power Transfer; EVs: Electric Vehicles Charging Efficiency; FEM: Finite Element Method; ME: Maxwell Equations; FM: Ferromagnetic; SMM: Soft Magnetic Material; SS: Secondary Series

\section{Introduction}

Over the past decades, there has been a growing tendency for developing environment friendly energy usage all over the world due to the increasing concerns on the reduction of conventional fossil fuels, environment pollution and the global climate change issues, for which the development progress on electric vehicles (EVs) and hybrid electric vehicles (HEVs) has been driven rapidly forward. EVs have shown its advantages on addressing most of the environmental challenges while reliability and safety concerns of the traditional plug-in EVs are still obstructions. Whereas, the contactless power transfer (CPT) technology has capabilities to allow eliminations of cables, slip rings and exposed plugs, which could increase the charging safety with no sparkling and the reliability under extreme environmental conditions.
Based on the wireless power transmitting (WPT) proposed by Nikola Tesla, the fundamental principles of Faraday's law of induction and Maxwell's equations [1,2] in the $19^{\text {th }}$ century, the contactless power transfer (CPT) using loosely coupled inductive energy transfer or electromagnetic resonant coupling could show the main advantages such as wireless charging convenience and charging implementation safety especially in dusty or humid conditions. Numerous studies and industrial applications worldwide have been carried out in the past two decades. A 2-kW circular pads based inductive power transmitting (IPT) model with 700$\mathrm{mm}$ diameter windings and 200-mm charging air gap was constructed and tested in 3D FEM software in [3] , which examined that this circular pad modular can result in satisfactory outputs 
and that the result difference between the simulation and experiment tests was claimed within $10 \%$ despite no detailed discussions about RMS real power ratings. In order to cancel out the reactive power generation from inductive coupling system to increase the RMS real power to the load end, four basic capacitor compensation topologies have been introduced and studied, which are primary series to secondary series (SS), primary series to secondary parallel (SP), primary parallel to secondary series (PS) and primary parallel to secondary parallel (PP) circuits. In [4], a current source-based SP compensation IPT system was built, resulting in an output of $200 \mathrm{~W}$ at $100 \mathrm{kHz}$ with up to $88 \%$ excluding inverters. An innovative primary series-parallel to secondary series (SPS) combination compensation topology was proposed in a large sized IPT system with 15-cm air gap [5] and was claimed to show a high tolerance to misalignment degree.

To allow voltage source based inverters to produce constant AC current from the primary side of a CPT system, an inductor-capacitor-inductor (LCL) network was embedded with an SP compensation, which was reported to achieve a constant current waveform independent of the load end value variations [6] and to minimize the reactive currents in the pickup coil [7].

A larger dimension stationary CPT system model in laboratory was designed in [8] with width of $200 \mathrm{~cm}$ and length of $100 \mathrm{~cm}$, which was reported to acquire an output power of $15 \mathrm{~kW}$ with an air gap of $15 \mathrm{~cm}$ and a lateral displacement tolerance of $40 \mathrm{~cm}$. The study in [9] compared the four compensation performances with a 2-kW model and suggested that the SS compensation method has lower copper loss than the other three methods. To improve power transfer capability and efficiency, a dual side control IPT scheme was proposed in [10] based on decoupling and primary track current control [11], validating a 7\% efficiency improvement with 813-mm diameter circular power transmitting pads and each winding of 1300 strands.

In this paper, to address the main challenges of maximizations of the system efficiency, power transfer ratings, and air gaps of coupling coils, the H-Shaped magnetic couplers with a series-to-series (SS) compensation has been fabricated and imple- mented. By analysis and comparison on the experimental results and system performance, firstly, the improvements of the flux linkage, magnetic flux density and flux line distribution can be noticed due to the contributions of using soft magnetic material core Steel 1010 and the effectiveness of its high permeability for the overall system electromagnetic performance enhancement. Secondly, the specifically designed H-shaped cores could be of significant help on facilitating magnetic strengths at critical points in the formed inductive electromagnetic field by shaping the flux line trajectories, which, from the perspective of electromagnetics, utilizes the most of the stored magnetic field energy leading to higher induced currents and magnetic fluxes through the secondary coil.

Thirdly, it can be found that the maximum system efficiency and power transfer to load could be reached when the system operating frequency is set close to, but not exactly to, the calculated resonant frequency of the primary coil. Nevertheless, in some cases of near-field coupling such as the models with $10-\mathrm{mm}$ and 20-mm coupling distances in this paper, the maximum system efficiency and power rating can be achieved twice at two peaks, respectively, which could be caused by the combined effects of the superposed fields from two coils and by the actual real-time power factors (PF) resulting in various RMS real powers. Furthermore, as the secondary coil resonant frequency is increasing in terms of higher strengthened coupling, approximating the system operating frequency to the calculated secondary coil resonant frequency may contribute to the second peak values of efficiency and power.

\section{System Overview}

\section{System Structure}

Regarding the actual overall performance of a CPT system and the electromagnetic field characteristics generated in real world applications, the inductive coupling modular could play a significant role whilst the wireless energy transmitting phenomenon occur due to the contributions of the continual magnetic flux generations and the effectiveness of inductive coupling within the non-linear ferromagnetic core materials and in between the coupler coils.

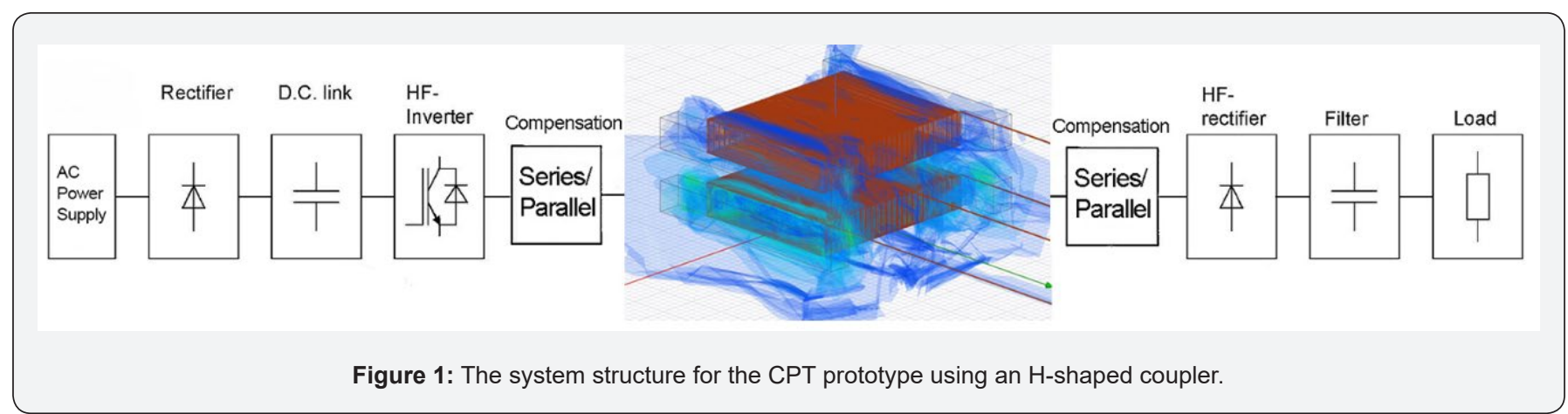

By optimally shaping the flux line trajectories and forming the flux distributions using $\mathrm{H}$-shape soft magnetic cores, the actual flux linkages and induced currents can be indices to enhance the inductive coupling performance in real-world CPT scenarios. Specifically, the high permeability and low core loss of soft ferromagnetic materials could contribute to flux linkage and magnetic 
flux density in order for the CPT system to produce an optimized electromagnetic field performance especially when the electromagnetic resonant coupling tends to occur at specific conditions [12]. A stationary CPT system layout using the designed H-shape coupler with four compensation topologies (series-to-series, series-to-parallel, parallel-to-series and parallel-to-parallel) has been shown in Figure 1. As presented in the CPT system in Figure 2 , a series-to-series (SS) compensation has been selectively adopted to implement the investigations in this paper.
To analyze the inductive coupling performance of the proposed CPT system, based on the RLC circuit theories, the impedance matching principle and the initial system tests with calculations for conditions of approaching electromagnetic resonant coupling, the designed system circuit parameters have been configured in Figure 2, in which both the compensation capacitors are set with $150 \mathrm{nF}$, the load is set with a resistor of $50 \mathrm{ohm}$ and the a voltage source with an RMS value of $5 \mathrm{kV}$ has been adopted as the system power supply

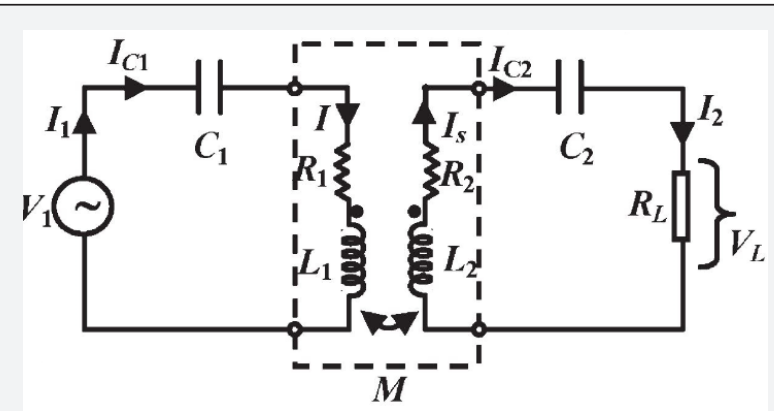

Figure 2: The simplified equivalent circuit with SS compensation topology.

\section{Geometric Design for Couplers}

As mentioned above, the particularly shaped soft ferromagnetic core with analytical geometry design and characteristics of high permeability can be able to form the magnetic flux paths as expected in order to facilitate the generations of flux linkage, magnetic flux density and field strength. Therefore, the Steel 1010 with a relative permeability $\boldsymbol{\mu}$ of 1000 is employed as the ferro- magnetic core material in this coupling modular built in the software package ANSYS 3D Maxwell as shown in Figure 3. Besides, considering the advantages of proximity effects mitigation, skin effects reduction and high frequency application capability, Litz wire is adopted for the windings in this prototype. The geometric model design parameters of the small sized magnetic coupler have been presented in Table 1 below.

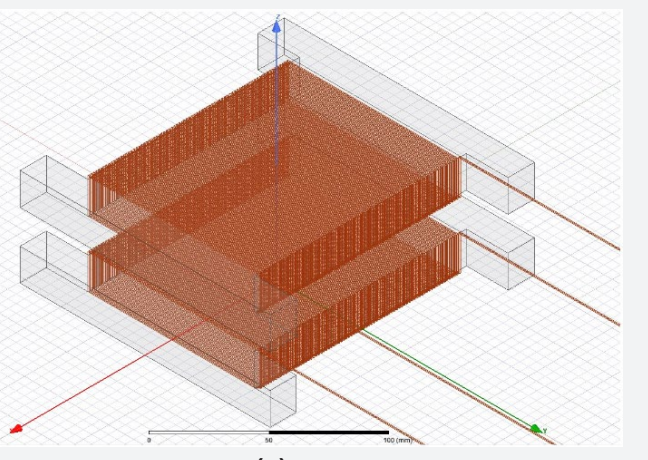

(a)

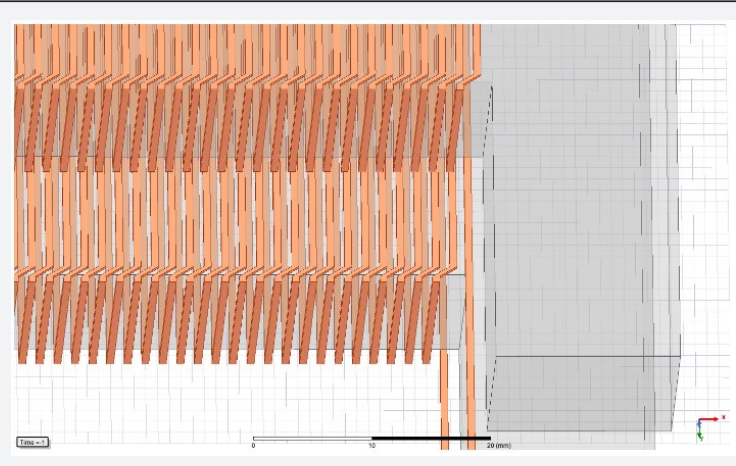

(b)

Figure 3: The H-shape coupler prototyped in 3D FEM environment.

(a) An overview of the coupler.

(b) Details of windings.

Table 1: The geometric specifications of the designed H-shape couplers for the CPT system.

\begin{tabular}{|c|c|}
\hline Parameters & Values \\
\hline Winding size & $100 \mathrm{~mm} * 100 \mathrm{~mm} * 20 \mathrm{~mm}$ \\
\hline Soft magnetic core size & $150 \mathrm{~mm} * 150 \mathrm{~mm} * 20 \mathrm{~mm}$ \\
\hline H-shaped core bar size of each side & $150 \mathrm{~mm} * 15 \mathrm{~mm} * 20 \mathrm{~mm}$ \\
\hline Primary winding number of turns & 80 turns \\
\hline Secondary winding number of turns & 80 turns \\
\hline Air gaps of the CPT charging system & $10 \mathrm{~mm}, 20 \mathrm{~mm}$ and $30 \mathrm{~mm}$ \\
\hline
\end{tabular}




\section{Theoretical Basics and Numerical Representations}

In order to investigate and analyze the CPT system outputs with its expected electromagnetic coupling performance, the electric current density J induced on the secondary side of the coupler model is required to be determined. Hence, the Maxwell equations constitutively expressed by Ampere's law in Eq. (1), Gauss' law in Eq. (2), Faraday's law in Eq. (3) and the B-H curve relation in Eq. (4) are required to be solved:

$$
\begin{gathered}
\Delta \times \mathrm{H}=\mathrm{J}(1) \\
\nabla . B=0(2) \\
\Delta \times E=\frac{-\partial B}{\partial t}(3) \\
B=\mu H(4)
\end{gathered}
$$

where $\mathrm{H}$ is the magnetic field strength in ampere per meter $(\mathrm{A} / \mathrm{m}), \mathrm{B}$ is the magnetic flux density in tesla $(\mathrm{T}), \mathrm{E}$ is the electric field strength in volt per meter $(\mathrm{V} / \mathrm{m})$, and $\boldsymbol{\mu}$ is the permeability non-linearly depending on local value of $B$ in $B=f(H)$. The electric current density $\mathrm{J}$ is in ampere per square meter $\left(\mathrm{A} / \mathrm{m}^{\wedge} 2\right)$.

$$
B=\Delta \times A(5)
$$

Simultaneously with Ohm's law in electromagnetics by Eq. (6) and material equations of Pouillet's law by Eq. (7), the 3D finite-element method (FEM) is adopted to numerically solve Maxwell equations above and the relative material equations, using the methods of discretizing the modelled 3D space by tetrahedrons, translating the differential equations into algebraic equations [13]. The methods of semi-iterative conjugate gradient for each magnetic vector potential A [14], based on the initial B-H curve, constant values of $\boldsymbol{\mu}$ can be selected for each finite element depending on local nonlinear value of B for the beginning. The flux density $B$ values afterwards can be computed according to the calculated magnetic vector potential A by Eq. (5) derived from Gauss' law of Eq. (2). Repeatedly, the numerical computations can be completed until the convergence of the element permeability.

$$
\begin{gathered}
J=\sigma E(6) \\
R=\rho \frac{1}{C}(7)
\end{gathered}
$$

where $\sigma$ is the material-dependent parameter conductivity. $\rho$ is the resistivity of the windings. $R$ is known as electric resistance of the wire material. $l$ is the total length of the wire. $C$ is the cross-sectional area of the wire. Based on the equations above, Eq. (1) turns into:

$$
\begin{gathered}
\Delta \times\left(\frac{1}{\mu} \Delta \times A\right)=J(8) \\
E=\nabla \phi-\frac{\partial A}{\partial t}(9)
\end{gathered}
$$

together with Faraday's law and Lorenz gauge vector potential by Eq. (9) [15], by using 3D FEM computations, the magnetic flux density $\mathrm{B}$, the electric field strength $\mathrm{E}$ and the magnetic field with values of magnetic flux $\phi$ in weber can be numerically solved and determined. In order to determine the self-inductance values of $\mathrm{L}$ in henry produced by the electromagnetic field on the both sides of the CPT system, the relations between the coil current values of $i$ and the flux linkage $\lambda$ satisfy:

$$
\begin{gathered}
\lambda_{1}=N_{1} \phi_{1}=L_{1} i_{1}(10) \\
\lambda_{2}=N_{2} \phi_{2}=L_{2} i_{2}(11) \\
L=\frac{\lambda}{i}
\end{gathered}
$$

In addition, based on the RLC circuit theories [16] and circuit resonance conditions, the approximate natural resonant frequency for the designed coupling system in this paper could be determined in order to specify the power source operating frequencies, which then is for the analysis and optimization of the actual system performance. To study the actual inductive magnetic coupling outcomes and electromagnetic field performance represented and reflected by the numerical vectors and scalars above, the actual RMS power generations given by Eq. (13) and the efficiencies given by Eq. (14) and (15) from the front end to the load end of the CPT system are required to be calculated, compared and analyzed. $\cos \varphi$ is the power factor (PF) caused by phase difference $\boldsymbol{\varphi}$ between induced voltage and current waveforms.

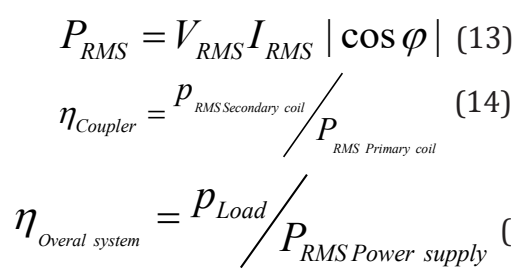

\section{Results and Analyses}

Flux linkage and currents based on electromagnetic field performance0ptimal flux linkage and currents with a 10-mm air gap based on electromagnetic metrics: To investigate the actual electromagnetic field system performance of the CPT coupler proposed, the flux linkage $\lambda$ and coil current $i$ are required to be addressed. Based on Faraday's law of induction in electromagnetics and Eq. (10-12) above, the changing magnetic flux $\boldsymbol{\phi}$ that causes electromotive force (EMF) and flux linkage $\lambda$ is determined by the electric field strength $\mathrm{E}$ and the magnetic field density B. Thus, analyzing the actual changing status of the system in electromagnetic field in Figure 4 and observing the flux linkage waveforms in Figure 5, with reference to the induced current waveforms in Figure 6, can be proper methods to evaluate an optimal CPT system's characteristics with a 10-mm air gap at about the natural resonant frequency of the specific prototype. As presented in Figure 4, by the effects of flux line forming of the 
H-shape cores, both the vector arrows of magnetic flux density B and magnetic field strength $\mathrm{H}$ tend to be able to reach their peak magnitude values at the edged corner of the very end points of the $\mathrm{H}$-shape cores, with maximum scalar values of $9.364 \times 10^{\wedge}(-1)$ Tesla and $1.2608 \times 10^{\wedge} 5 \mathrm{~A} / \mathrm{m}$, respectively From Figure 5, it can be found that both the alternating flux linkage waveforms of two coils tend to be stable sinusoidal AC waveforms since about $0.5 \mathrm{~ms}$ onwards, which means the CPT system electromagnetic stability is able to be immediately established after only 4 periods.

Once the outputs tend to be AC stable, the amplitudes of the flux linkages have been measured to be $0.1044 \mathrm{~Wb}$ and $0.1196 \mathrm{~Wb}$ for the primary coil and secondary coil, respectively, which are corresponding to RMS values of $0.0738 \mathrm{~Wb}$ and $0.0846 \mathrm{~Wb}$.

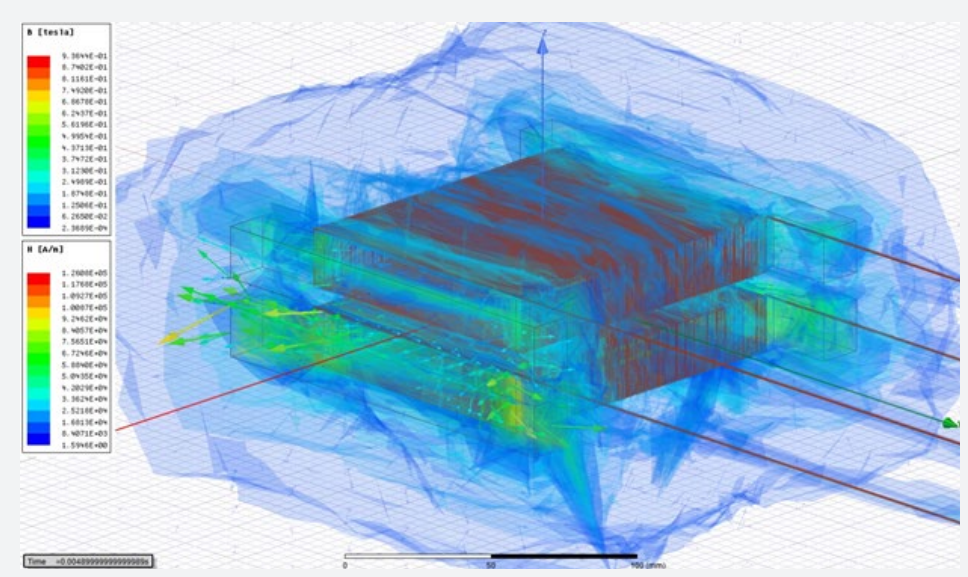

Figure 4: Electromagnetic field overlays of the CPT coupler with an air gap of $10 \mathrm{~mm}$, at $8000 \mathrm{~Hz}$.
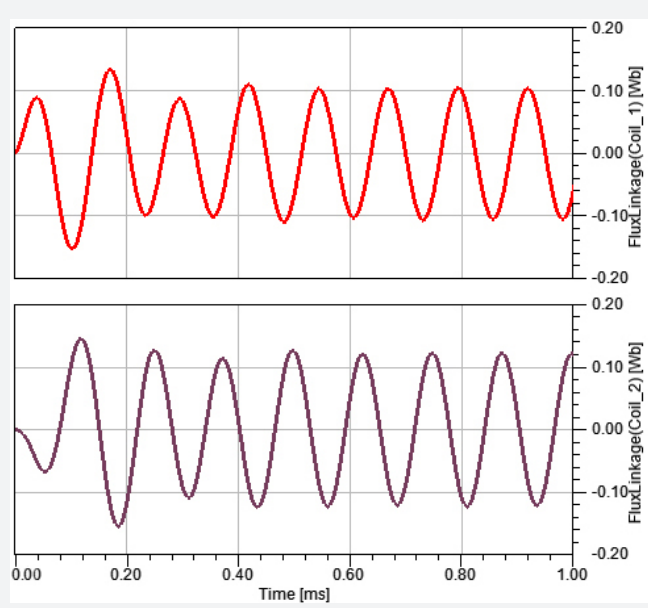

Figure 5: Flux linkage waveforms of the 10-mm air gap CPT system at $8000 \mathrm{~Hz}$.

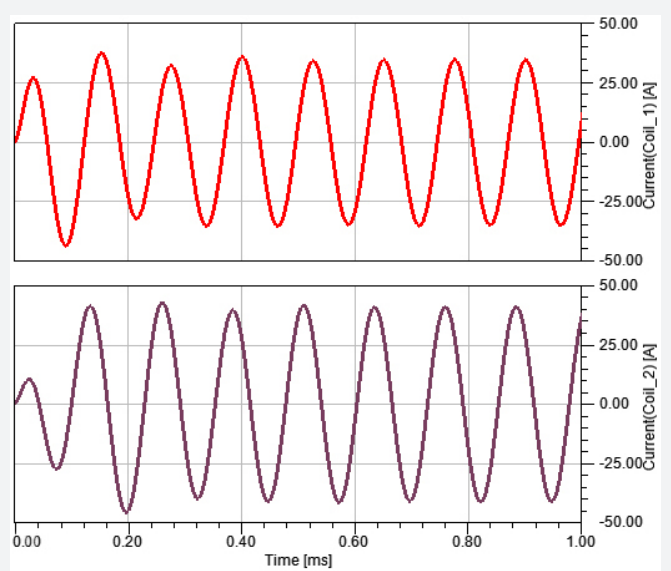

Figure 6: Current waveforms of the 10-mm air gap CPT system at $8000 \mathrm{~Hz}$. 
From the current waveforms presented in Figure 6, similarly, both the primary coil and secondary coil currents tend to be AC stable after 4 periodical waveforms, reaching amplitudes of 34.5679 $\mathrm{A}$ and $41.7339 \mathrm{~A}$, corresponding to RMS values of $24.4432 \mathrm{~A}$ and 29.5103 A, respectively. Based on the Figures 5\&6, compared to the longtime of a full charging cycle for the EV batteries, $0.5 \mathrm{~ms}$ is time transient and negligible. In other words, this 10-mm air gap $\mathrm{H}$-shape CPT system could accomplish a fast-inductive coupling matching towards constant stable AC power transfer for EVs once the vehicle receiving side is started for CPT charging. Besides, it can be noticed that the RMS flux linkage of the secondary coil is higher than the primary coil by $0.0108 \mathrm{~Wb}$ and the RMS current of the secondary coil is 5.0671 A higher than the primary coil as well, which could be mainly due to the contributions of SS compensation capacitors and the alternating field energy stored in the space since the initial coupling of about $0.3 \mathrm{~ms}$. Nevertheless, when considering the phase differences of actual real-time voltages and currents on both sides, the calculated RMS power of the secondary side is lower than the primary side due to the power factor (PF) $\cos \boldsymbol{\varphi}$ corresponding to the reactive power's $\sin \varphi$ that is for the continually establishing and sustaining the optimistic electromagnetic field of the CPT system.

Analysis on flux linkages and currents for different air gaps at various operating frequencies: In this investigation, the H-shape coupler CPT prototypes with 10, 20- and 30-mm air gaps, across various ranges of operating frequency, have been implemented and analyzed. The system performances at electromagnetic resonant coupling conditions have also been emphasized in each model by peak values and characteristic trends. From Figure 7 , it could be seen that the secondary coil current could have two peak values at $8 \mathrm{kHz}$ and $11 \mathrm{kHz}$, which are $29.57 \mathrm{~A}$ and $29.52 \mathrm{~A}$, respectively. The primary coil current reaches its maximum value of $33.836 \mathrm{~A}$ at the operating frequency of $11 \mathrm{kHz}$. However, both the primary and secondary flux linkages show decreasing tendencies when the operating frequency supply increases although there is one salient point with value of $0.070 \mathrm{~Wb}$ for the primary linkage at $11 \mathrm{kHz}$. For the 20-mm air gap CPT model, in Figure 8, both the primary and secondary coil currents tend to have stably high values between 7 to $10 \mathrm{kHz}$ while the secondary coil current shows its maximum value of $25.23 \mathrm{~A}$ at $8 \mathrm{kHz}$ and the primary coil current reaches its maximum of 28.24 at $9 \mathrm{kHz}$. It can be seen from Figure 8 that the values of secondary flux linkage could stay optimistic when the operating frequency is between 7 to $8 \mathrm{kHz}$. In Figure 9, it could be seen that the secondary coil current reaches its maximum of $20.29 \mathrm{~A}$ at $8 \mathrm{kHz}$ where the primary coil current almost drops to its bottom value. There is a maximum salient point at $6 \mathrm{kHz}$ for the secondary flux linkage however the primary flux linkage shows two sharp knee points at 6 and $7 \mathrm{kHz}$.

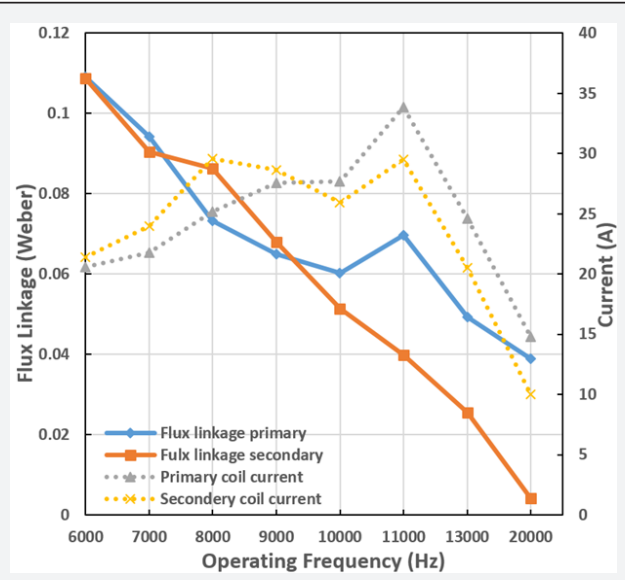

Figure 7: Flux linkages, currents vs operating frequencies for the 10-mm air gap model.

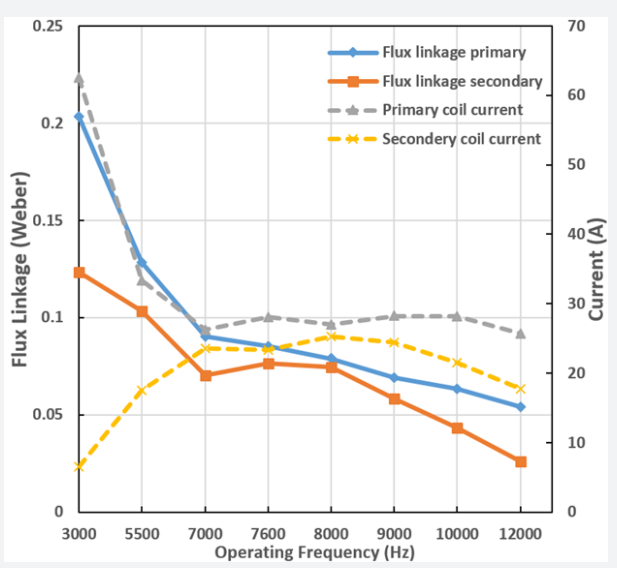

Figure 8: Flux linkages, currents vs operating frequencies for the 20-mm air gap model. 


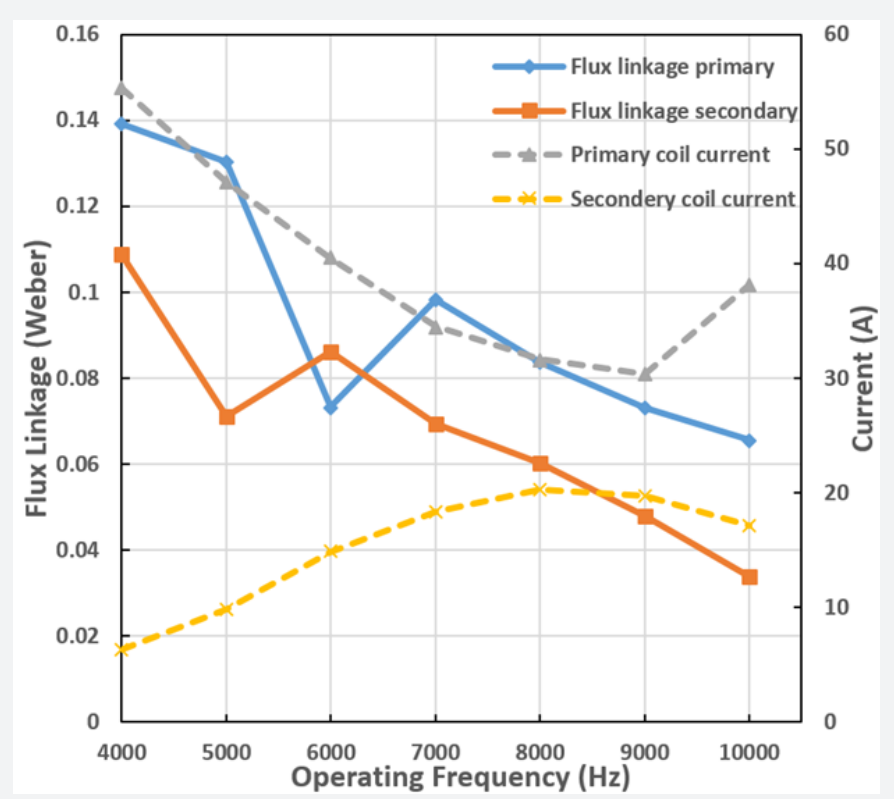

Figure 9: Flux linkages, currents vs operating frequencies for the 30-mm air gap model.

\section{Real Powers and Efficiencies}

Due to the different power factors (PF) and various electromagnetic field parameters in the CPT models with changeable air gaps, the RMS real power generations on each modular of the system could be different, which can lead to different efficiency on every two elements selected for analysis. In the 10, 20- and 30$\mathrm{mm}$ air gap CPT models, the coil transmitting efficiency, the overall system efficiency from the power source front end to the load rear end have been analyzed and compared in Figures 10-12. In each figure, the system input power, primary coil power, the secondary coil power and the system output power against the operating frequency have also been presented. As can be seen from Figure 10 , both the system efficiency and the coil efficiency show the two peak values at two operating frequency points. The system efficiency reaches $57.407 \%$ at $7 \mathrm{kHz}$ and $59.534 \%$ at $11 \mathrm{kHz}$. The coil efficiency reaches $79.277 \%$ at $7 \mathrm{kHz}$ as well and $83.806 \%$ at 10 $\mathrm{kHz}$. The overall system output power RMS values on load could stay satisfactory from $8 \mathrm{kHz}$ to $11 \mathrm{kHz}$, with a maximum value of $42.812 \mathrm{~kW}$ at $8 \mathrm{kHz}$.

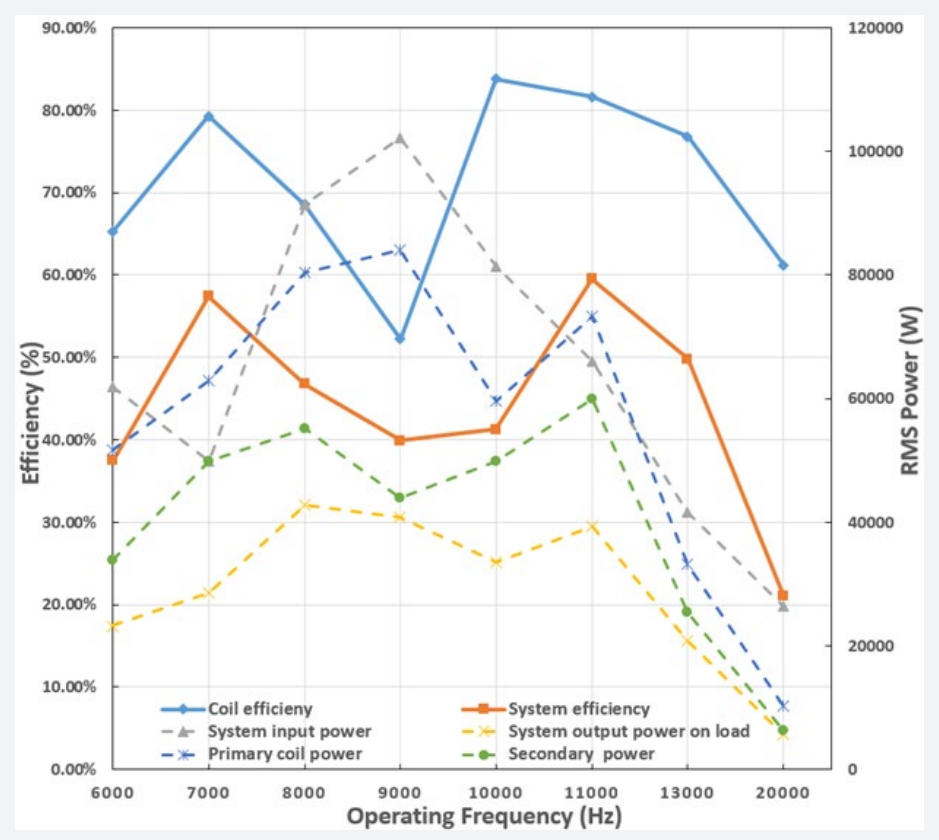

Figure 10: Efficiencies, RMS real powers vs operating frequencies for the 10-mm air gap model. 


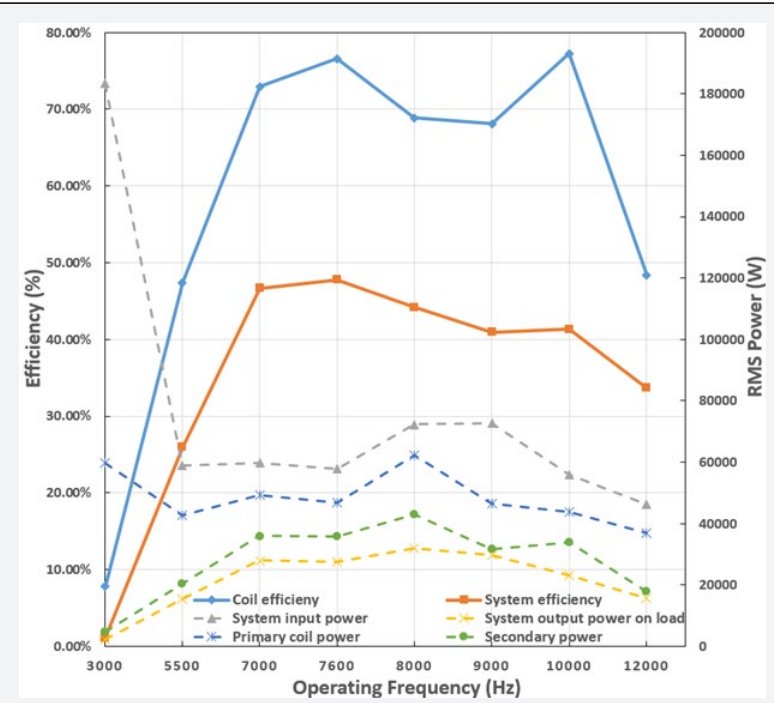

Figure 11: Efficiencies, RMS real powers vs operating frequencies for the 20-mm air gap model.

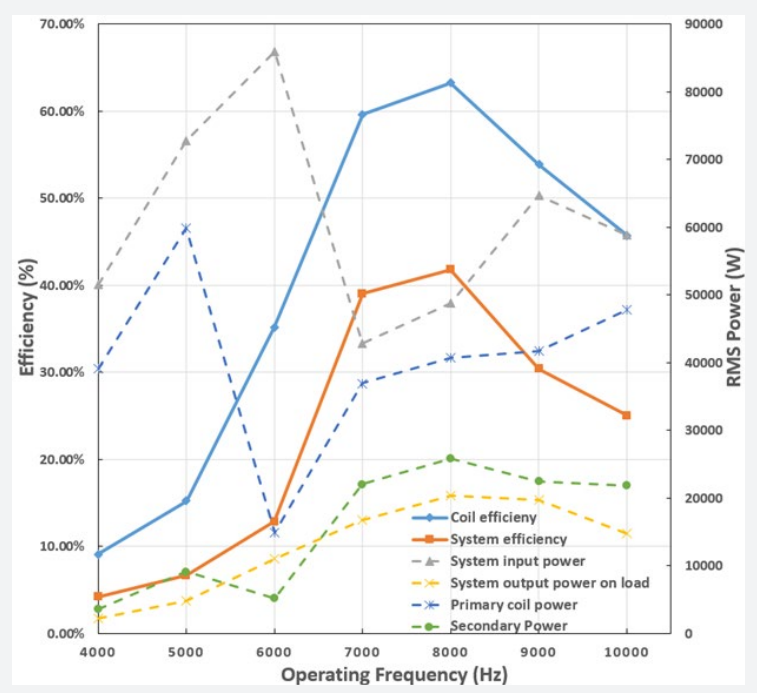

Figure 12: Efficiencies, RMS real powers vs operating frequencies for the 30-mm air gap model.

As shown in Figure 11, the 20 -mm air gap system can produce stably high coil efficiency over $68 \%$ across the operating frequency range of $7 \mathrm{kHz}$ to $10 \mathrm{kHz}$, with a maximum point of $77.220 \%$ at $10 \mathrm{kHz}$. The system overall efficiency shows a wide range of high efficiency over $40 \%$ across the operating frequency of $7 \mathrm{kHz}$ to 10 $\mathrm{kHz}$, with a peak value of $47.754 \%$ at $7.6 \mathrm{kHz}$. It also can be seen that the system output power to the load achieve its maximum of $31.954 \mathrm{~kW}$ at $8 \mathrm{kHz}$. The secondary coil and the system load could show stable real power generations within a wide range of operating frequency in Figure 11. It can be found, from Figure 12, that the system efficiency and coil efficiency share almost the same trend against the operating frequency. The coil efficiency reaches its peak value of $63.233 \%$ at $8 \mathrm{kHz}$, at which the system efficiency reaches its maximum of $41.776 \%$. Similarly, both the secondary coil power and the output power on load achieve their maximum values of $25.753 \mathrm{~kW}$ and $20.398 \mathrm{~kW}$, respectively, at the operating frequency of $8 \mathrm{kHz}$.

\section{Calculated Natural Resonant Frequencies and Calculated Inductances}

As found and concluded in [12], the natural resonant frequencies of primary side and secondary side of the CPT system could be varied directly depending on the generated inductance $\mathrm{L}$, based on. $f_{\text {resonant }}=\frac{1}{2 \Pi \sqrt{L C}}$ Fundamentally, the magnetic flux $\boldsymbol{\phi}$ and flux linkage $\lambda$ in Eq. (9-12) are subject to the actual coupler's geometric design, which then determines the inductances on both coils. Therefore, it is necessarily required to investigate and analyze the relations between the increasing operating frequencies and the changeable resonant frequencies of primary and secondary sides by tracking and approximating the natural resonant frequency using various operating frequencies, in addition to the different air gaps against the system performance parameters in sections above. From Figure 13, it can be noticed that the primary side's calculated natural resonant frequency tends to fall within a range of 8.2 to $8.5 \mathrm{kHz}$, which means the natural resonant frequency of 
the primary side could be independent of increase of the operating frequency supply when the CPT system air gap is $10 \mathrm{~mm}$.

This could be due to the stably flat values of calculated inductance of the primary side at about $0.0024 \mathrm{H}$, across the operating frequency of 8 to $13 \mathrm{kHz}$. On the other hand, the electromagnetic resonant coupling for optimized CPT system outputs could be approximated by feeding the operating frequency of 8.2 to $8.5 \mathrm{kHz}$, which has also been observed by the flux linkage, induced current, RMS power and system efficiency values in previous sections. However, compared with the primary side, the calculated natural resonant frequency of the secondary side keeps increasing versus the operating frequency, with nearly a proportional relation of a gradient of 1 , which could be mainly caused by the constantly decreasing inductance of the secondary side, based on Figure 13.
CPT prototype. it can be found from Figure 14 that the calculated resonant frequency of the primary side tends to achieve stable values of around $7.5 \mathrm{kHz}$. Whereas, the calculated resonant frequency of the secondary side keeps rising, in terms of feeding higher operating frequency. Regarding the calculated inductance values of two sides, the primary side's calculated inductance tends to be stable at about $0.0028 \mathrm{H}$ whilst the secondary side's calculated inductance keeps gradually decreasing when the system operating frequency is set growing over $12 \mathrm{kHz}$. In Figure 15, it can be seen that there exists a knee point at $6 \mathrm{kHz}$ for the curve of calculated resonant frequency of primary side, which could be caused by the dramatic dropped value of primary coil flux linkage in Figure 9. And the calculated resonant frequency of primary side tends to stabilize at $6.7 \mathrm{kHz}$. Similar to the trend in Figure 14, the secondary side's calculated resonant frequency keeps increasing due to constantly descending inductance.

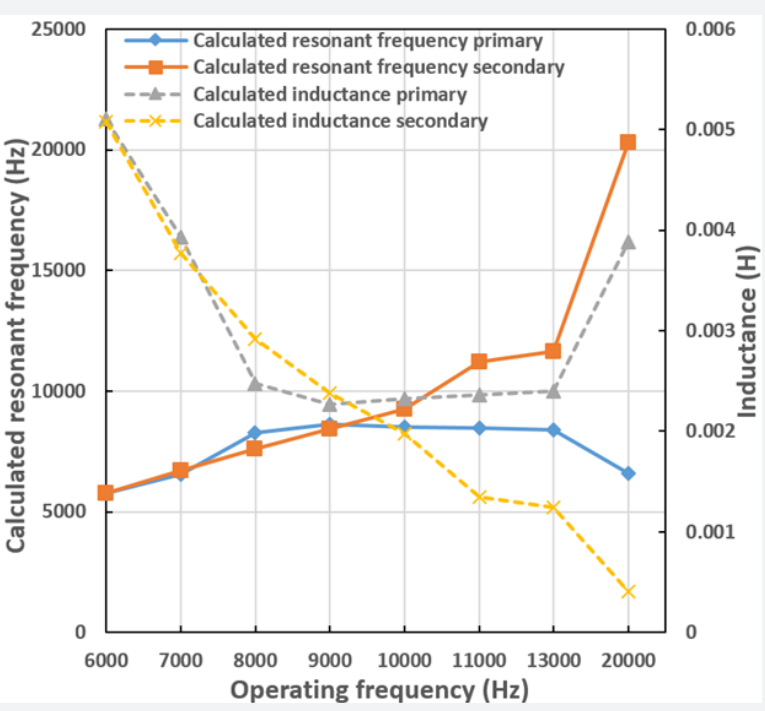

Figure 13: Calculated resonant frequencies, calculated inductances vs operating frequencies for the 10-mm air gap CPT prototype.

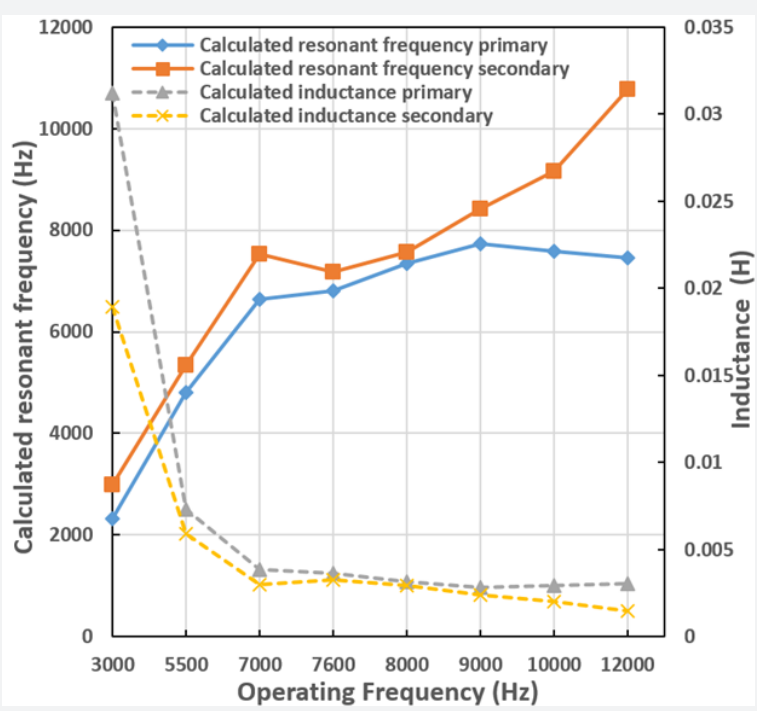

Figure 14: Calculated resonant frequencies, calculated inductances vs operating frequencies for the 20-mm air gap CPT prototype 


\section{Juniper Online Journal Material Science}

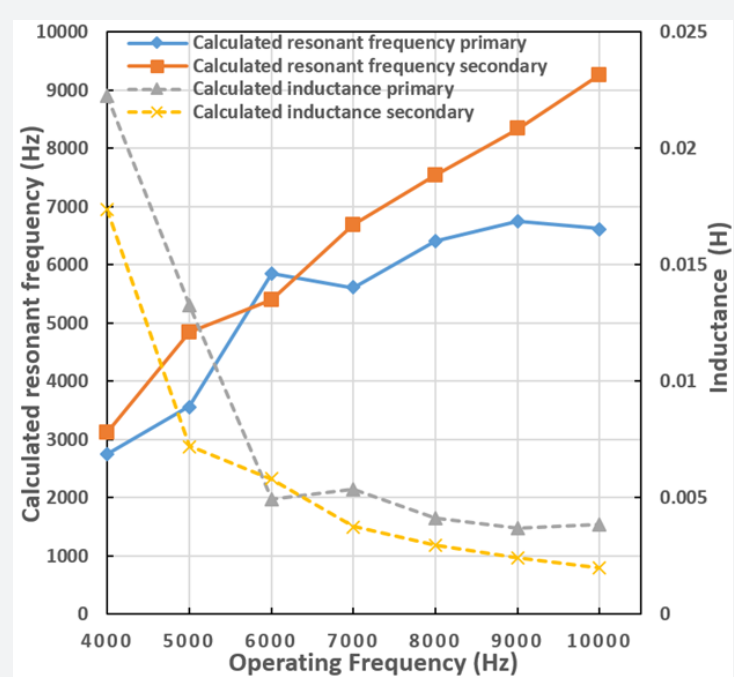

Figure 15: Calculated resonant frequencies, calculated inductances vs operating frequencies for the 30-mm air gap CPT prototype.

\section{Discussion}

From the perspectives of electromagnetics, due to the positive contributions of employing ferrite material with high permeability and H-shape effects, the designed CPT model has shown the improvements on the flux linkage, magnetic flux density, magnetic field strength and flux line distribution based on the analyses in the previous sections. Especially, the specifically designed H-shape soft ferromagnetic cores have demonstrated its significance on facilitating the magnetic strength and flux density at critical points and positions of the couplers such as the external edges and corners of the $\mathrm{H}$-shape cores with expected maximum scalar values and vector directions representing the optimally formed flux distributions and trajectories.

Importantly, the maximum system efficiencies and maximum power transfer ratings of each model coupling scenario can be achieved by approximating the operating frequency to the range of calculated natural resonant frequencies of the primary side. In other words, all the CPT models tend to have an approximate resonant frequency range for the primary side rather than the secondary, as shown in Figures 13-15, the optimal system performance including the maximum system efficiencies and maximum power transfer ratings can be reached when the system tends to perform at magnetic resonant coupling although there would be necessary trade-offs between the optimal efficiency and the optimal power ratings within the corresponding frequency range for electromagnetic resonance in each model as these two optimal values may not occur at the same time.

After comparing the proposed three models, obviously the 10-mm air gap CPT system demonstrates the best coupling performance with the strongest magnetic strength and flux density. In the meantime, the $10-\mathrm{mm}$ air gap system shows two optimal system efficiency points not only at its original calculated natural resonant frequency of about $7 \mathrm{kHz}$ of the primary side, but also at $11 \mathrm{kHz}$, which may be a consequence caused by the combined effects of the superposed fields induced from both coils, especially the highly strong resonant coupling from the secondary side. Besides, the positive effect from compensation capacitor and the lowest reactive power on the secondary side may occur at $11 \mathrm{kHz}$ to lead to highest system efficiency and RMS power to load for the secondary time during the wide range of frequency.

In terms of the RMS real powers, the phase differences between $\mathrm{AC}$ voltages and currents play a vital role in working out not only the real power for energy transfer to the load but also the reactive power for establishing and sustaining the electromagnetic field with stored field energy. Thus, the actual phase difference produced in real-time couplings is essentially to be studied and tuned when necessary by adjusting compensation capacitors to an optimal condition at the fixed optimal range of operating frequency. This would be a good topic for further investigations since the stored field energy contributes to the overall system performance and efficiency calculations. In order to further improve and optimize the flux distributions, the electromagnetic field shielding could be considered in the future research.

By using different shielding materials and shapes, the optimal designs towards electromagnetic field performance could be investigated. Furthermore, more potentially optimal core shapes could also be proposed and studied in the future research. Regarding the limitations, the model is supposed to be tested in terms of misalignment degrees in the future research. In reality, the lateral and longitudinal displacements caused in parking a vehicle could significantly affect the electromagnetic field generations and the quality of coupling, which indirectly leads to unsatisfactory losses and power ratings to the CPT system. In this paper, the misalignment tolerance has not been taken into account as a variable. In addition, the model size in this paper is not sufficiently large to be equivalent to a real application for a vehicle chassis due to the considerations on computational complexity and accuracy in the selected 3D FEM environment. The improved model size is supposed to be larger and scaled up in the future discussion. 


\section{Conclusion}

To address and achieve the maximizations of the system efficiency, the power transfer ratings, and the air gaps of coupling coils, a novel small sized CPT prototype using geometrically optimized H-shaped magnetic couplers and series-to-series (SS) compensation has been analytically modelled and investigated from prospective of electromagnetics, RMS power and efficiency. Three CPT models with 10, 20- and 30-mm air gaps have been investigated in this paper. It can be found that the 10 -mm air gap H-shape soft ferromagnetic coupler CPT system, as an optimal prototype, could produce a maximum system efficiency of 59.5\%, a coil transmitting efficiency of $83.8 \%$ and a maximum power output of $42.81 \mathrm{~kW}$ on the load end when the resonant coupling of CPT system tends to occur within a range of calculated resonant frequencies of the primary side. The enhancements of RMS flux linkage, magnetic flux density and magnetic field strength in terms of the actual electromagnetic performance produced have been demonstrated. In addition, the resonant couplings for the designed CPT systems relating to the calculated inductance and natural resonant frequency have been studied by result analyses and comparisons.

\section{References}

1. Grant I, Phillips W (1990) Electromagnetism (2 ${ }^{\text {nd }}$ edn), Manchester physics series, Chichester, UK.

2. Duffin WJ (1990) Electricity and Magnetism ( $4^{\text {th }}$ edn), McGraw-Hill, London, UK.

3. Budhia M, Covic G, Boys J (2011) Design and Optimization of Circular Magnetic Structures for Lumped Inductive Power Transfer Systems. IEEE Transactions on Power Electronics 26(11): 3096-3108.

4. Sohn Y, Bo H Choi, Eun S Lee, Gyu Lim, Gyu-Hyeong Cho, et al. (2015) General Unified Analyses of Two-Capacitor Inductive Power Transfer Systems: Equivalence of Current-Source SS and SP Compensations. IEEE Transactions on Power Electronics, 30(11): 6030-6045.

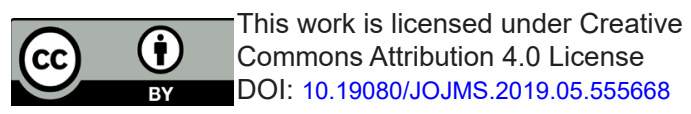

5. Villa J, Sallan J, Sanz Osorio J, Llombart A (2012) High-Misalignment Tolerant Compensation Topology for ICPT Systems. IEEE Transactions on Industrial Electronics, 59(2): 945-951.

6. Kissin M, Chang-Yu Huang, Covic, Boys (2009) Detection of the Tuned Point of a Fixed-Frequency LCL Resonant Power Supply. IEEE Transactions on Power Electronics 24(4): 1140-1143.

7. Keeling N, Covic G, Boys J (2010) A Unity-Power-Factor IPT Pickup for High-Power Applications. IEEE Transactions on Industrial Electronics 57(2): 744-751.

8. Choi S, Huh J, Lee W, Rim C (2014) Asymmetric Coil Sets for Wireless Stationary EV Chargers with Large Lateral Tolerance by Dominant Field Analysis. IEEE Transactions on Power Electronics 29(12): 6406-6420.

9. Sallan J (2009) Optimal Design of ICPT Systems Applied to Electric Vehicle Battery Charge. IEEE Transactions on Industrial Electronics 56(6): 2140-2149.

10. Wu H, Gilchris A, Sealy K, Bronson D (2012) A High Efficiency 5 kW Inductive Charger for EVs Using Dual Side Control. IEEE Transactions on Industrial Informatics 8(3): 585-595.

11. Covic G, Boys J, Tam A, Peng J (2008) Self-tuning pick-ups for inductive power transfer. IEEE Power Electronics Specialists Conference 34893494.

12. Duan J, Wang W (2018) Electromagnetic Coupling Optimization by Coil Design Improvements for Contactless Power Transfer of Electric Vehicles. In Proceedings of the Future Technologies Conference (FTC). Advances in Intelligent Systems and Computing 881: 944-958.

13. Sadiku M (1992) Numerical techniques in electromagnetics, Boca Raton, USA.

14. Silvester P, Ferrari R (1990) Finite elements for electrical engineers ( $2^{\text {nd }}$ edn), USA.

15. Jackson JD, Okun LB (2001) Historical Roots of Gauge Invariance. Reviews of Modern Physics 73(3): 663-680.

16. Grover F (1946) Inductance Calculations: Working Formulas and Tables. Dover 9: 1.

\section{Your next submission with Juniper Publishers will reach you the below assets}

- Quality Editorial service

- Swift Peer Review

- Reprints availability

- E-prints Service

- Manuscript Podcast for convenient understanding

- Global attainment for your research

- Manuscript accessibility in different formats

( Pdf, E-pub, Full Text, Audio)

- Unceasing customer service

Track the below URL for one-step submission https://juniperpublishers.com/online-submission.php 